\title{
Extraktion und Trocknung von Krappwurzel-Farbstoff
}

\author{
Eckhard Meyer, Josef Schmadl, Stephan Abramowski
}

\section{Zum Stand des Wissens über Historie, Chemie und Verfahrenstechnik der Krappfarbstoffgewinnung}

Farbstoffe aus den Wurzeln der Rubia tinctorum und anderer Krapp-Sorten zählten über Jahrtausende zu den wichtigsten Textilfarbstoffen. Sie wurden jedoch - bis auf einige Spezialanwendungen - von den preisgünstigeren synthetischen Farbstoffen verdrängt. Ein neues Umwelt- und Gesundheitsbewußtsein der Menschen führt neuerdings dazu, daß Pflanzenfarbstoffe seit einigen Jahren in besonderen Anwendungsbereichen der Textilienfärberei wieder zunehmend im industriellen Maßstab Interesse finden.

Über die Zusammensetzung des Krappfarbstoffes weiß man erst seitdem moderne Analysetechniken verfügbar sind, daß er eine komplexe Mischung von mehr als 20 Einzelkomponenten aus der Klasse der Anthrachinonfarbstoffe umfaßt, die etwa 4 Gew. $\%$ der Wurzelmasse ausmachen [1]. Die einzelnen Komponenten dieser Mischung sind noch nicht restlos identifiziert und die Zusammensetzung ist deshalb noch nicht quantitativ bekannt. Zudem gibt es Unterschiede in der Zusammensetzung in Abhängigkeit von der Krappsorte. Hauptkomponente des Wirkstoffgemisches ist das Alizarin, das darin größtenteils in glykosidisch gebundener Form vorliegt. Es wurde 1869 erstmals von Graebe und Liebermann synthetisch hergestellt. Das typische KrappRot ergibt sich erst aus dem Zusammenspiel der ganzen Farbstoffpalette und ist deshalb nicht identisch mit dem Alizarinrot. Abb. 1 enthält das UV-Spektrum eines wässrigen Krapp-Auszuges (a) [2] im Vergleich mit einem Spektrum mehrerer Einzelkomponenten (b) [3]. Das Spektrum der Mischung (a) ist durch das dominierende Alizarin geprägt, andere Farbstoffe, wie z. B. Purpurin, sind im Spektrum aber auch zu erkennen. Im Wellenbereich unter $300 \mathrm{~nm}$ absorbieren auch andere organische Stoffe, wie z. B. Gerbstoffe und Eiweiße. Typischer für die Alizarinfarbstoffe ist der Bereich um $430 \mathrm{~nm}$ im sichtbaren Bereich. Deshalb wurde dieser Bereich für die photometrische Prozeßkontrolle der Extraktionsversuche ausgewählt.

Die Extraktion ist eine der ältesten Verfahrenstechniken [4]. Dennoch sind keine Extraktionstechniken bekannt, mit denen der Krapp-Wirkstoff in einer dosierfähigen Form, z. B. als lagerfähiges Konzentrat oder nach Trocknung durch konditionierfähiges Pulver, isoliert worden wäre. Traditionell wurde ein wässriger Absud der gemahlenen Wurzeln hergestellt und gleichzeitig oder unmittelbar danach als Färbeflotte in Büttentechnik be-
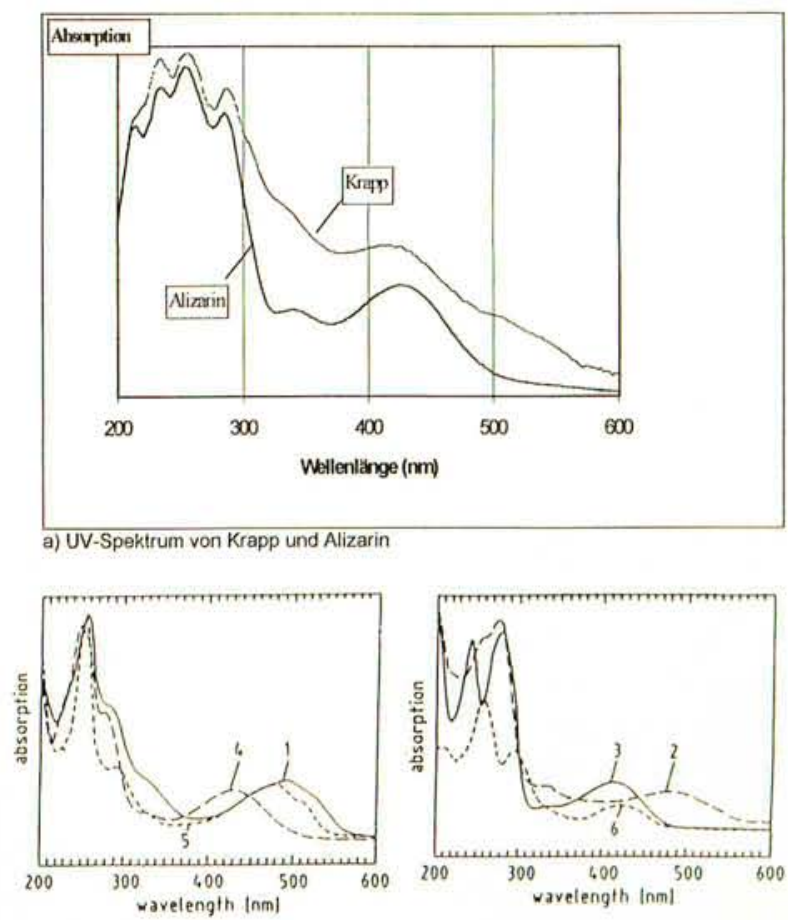

b) UV-Spektrum von Einzelfarbstoffen im Krapp (1 Pseudopurpurin, 3 Xanthopurpurin, 4 Alizarin, 5 Purpurin, 2 und 6 nicht identifiziert)

Abb. 1: Spektrum eines wässrigen Krapp-Extraktes (a) [2] im Vergleich zum Spektrum einiger Einzelkomponenten (b) |3|

nutzt. Das Trennen der Textilien von der Färbeflotte mit Wurzelresten machte aufwendige Reinigungsschritte erforderlich. Für heutige industrielle Färbeanlagen kommt diese Methode wegen der erforderlichen Verarbeitungspräzision, die eine exakte Dosierung erfordert, und wegen der nachfolgenden maschinellen Weiterverarbeitung nicht mehr in Betracht. Der Farbstoff sollte deshalb in lager- und dosierfähiger Konzentrat- oder Pulverform mit definiertem Farbstoffgehalt vorliegen. Verfahren zur Herstellung solcher Produkte, wie z. B. Extraktion, Eindampfung, Sprüh- und Gefriertrocknung, sind im Bereich der natürlichen Lebensmittelfarbstoffe (z. B. Rote Beete, Spinat) bereits Stand der Technik. Analoge Verfahren für pflanzliche Textilfarbstoffe, insbesondere fuir die Krapp-Farbstoffe, sind bislang nicht bekannt.

Ziel dieser Arbeit war es daher, verfahrenstechnische Auslegungsdaten für ein industrielles Verfahren zur Gewinnung von konditionerfähigem Krapp-Farbstoff durch Fest-flüssig-Extraktion, ggfs. Eindampfung und anschließende Trocknung zu gewinnen. Dabei sollte vor allem der Einfluß von Extraktionsparametern, wie Temperatur und Extraktionsdauer auf die Farbqualität untersucht werden. Es war zu überprüfen, inwieweit Emp- 
fehlungen aus traditionellen Färbeanleitungen (Temperatur maximal $75^{\circ} \mathrm{C}$ bei einer Belastungszeit von $120 \mathrm{~min}$ [5]) unter halbtechnischen Bedingungen Gültigkeit behalten. Zur Beurteilung der Qualität der Extrakte und Konzentrate wurden photometrische Extinktionsmessungen und standardisierte Probefärbungen (CIE- $L^{*} \mathrm{a}^{*} \mathrm{~b} \mathrm{~b}^{*}$-System $[2,6])$ herangezogen. Zum Trockungsverhalten wurden im Rahmen dieser Arbeit lediglich Vorversuche an verschiedenen Trocknertypen durchgeführt mit dem Ziel, ein geeignetes Trocknungsverfahren auszuwählen.

\section{Apparatur und Versuchsdurchführung}

In Abb. 2 ist die verwendete Extraktionsanlage schematisch dargestellt. Der eigentliche Extraktor ist ein 5 1-Behälter, der mit einem Teflonsieb (Maschenweite $0,3 \mathrm{~mm}$ ) ausgekleidet ist. Außerdem gehören zur Apparatur im wesentlichen eine 30 l-Vorlage B2 für frisches Extraktionsmittel, ein thermoölbeheizter 10 l-Verdampfer B1 mit DN50xH1000-Kolonnenaufsatz K1 und solegekühltem Brüdenkondensator W2 sowie Nachkühler W3. Fahrweisen als Perkolationsextraktion oder als Immersionsextraktion nach dem Soxleth-Prinzip sind wahlweise möglich. Außerdem kann die Apparatur unabhängig davon als mehrstufige Batch-Rektifikation und damit auch zum Aufkonzentrieren des Extraktes verwendet werden. Heizen und Kühlen erfolgen dabei mit Hilfe externer Kreisläufe, die hier nicht näher beschrieben sind. Zur Temperaturüberwachung sind im Extrak-tionskreislauf drei Pt100-Fühler eingebaut, die die Temperatur im Verdampfer, am Kolonnenkopf und im Destillat erfassen. Der Extraktionsmittelmengenstrom kann mit einem Schwebekörperdurchflußmesser fest eingestellt werden.

Ein Schema der Versuchsdurchführung mit beispielhaf- ten, typischen Zahlenwerten ist in Abb. 3 enthalten. Für die Extraktionsversuche wurde als Fahrweise der diskontinuierliche Kreuzstrom gewählt. Er ermöglichte es, Versuche mehrstufig und bei verschiedenen Temperaturen durchzuführen. Dazu wurde das Extraktionsmittel aus dem Verdampfer B1 mit Pumpe P1 über die Ventile V6 und V5 zunächst im Bypass außerhalb des Extraktors umgepumpt bis die Versuchstemperatur erreicht war. Dann wurde über Ventil V7 auf den Extraktor, in dem sich das Extraktionsgut in Form von zerkleinertem Krappwurzelmaterial befand, umgeleitet und der Extraktionsversuch gestartet. Während der Extraktion wurde die photometrische Extinktion des Extraktes gemessen. Der Versuch wurde als beendet betrachtet, sobald ein konstanter Höchstwert der Extinktion und damit die theoretische Trennstufe erreicht war. Abb. 4 zeigt einen typischen Extinktionsverlauf in Abhängigkeit von der Zeit bei drei verschiedenen Wellenlängen. Wegen der Vorbehandlung der Wurzeln mit Extraktionsmittel zwecks Quellung beginnen die Kurven nicht bei $\mathrm{E}=0$, sondern bei einem Anfangswert, der von der vorgelegten Extraktionsmittelteilmenge abhängig ist. Man sieht, daß bei diesem $60^{\circ} \mathrm{C}$-Versuch nach etwa 45 Minuten ein konstanter Wert der Extinktion erreicht wird.

Die meisten Versuche wurden dreistufig, in Einzelfällen auch ein- und sechsstufig durchgeführt, mit der Temperatur als Variationsparameter.

Der Extrakt wurde in der gleichen Apparatur aufkonzentriert, wobei Vakuumbetrieb nötig war, um die Extraktionstemperatur auch beim Aufkonzentrieren beizubehalten und damit eine Beeinträchtigung der Farbstoffqualität durch zu hohe Temperaturbelastung auszuschließen.

Die Trocknung erfolgte teilweise kontinuierlich in einem Labor-Rotationsverdampfer (Trocknung und Eindampfung in einem Schritt) und in einem Sprühtrockner Typ Büchi

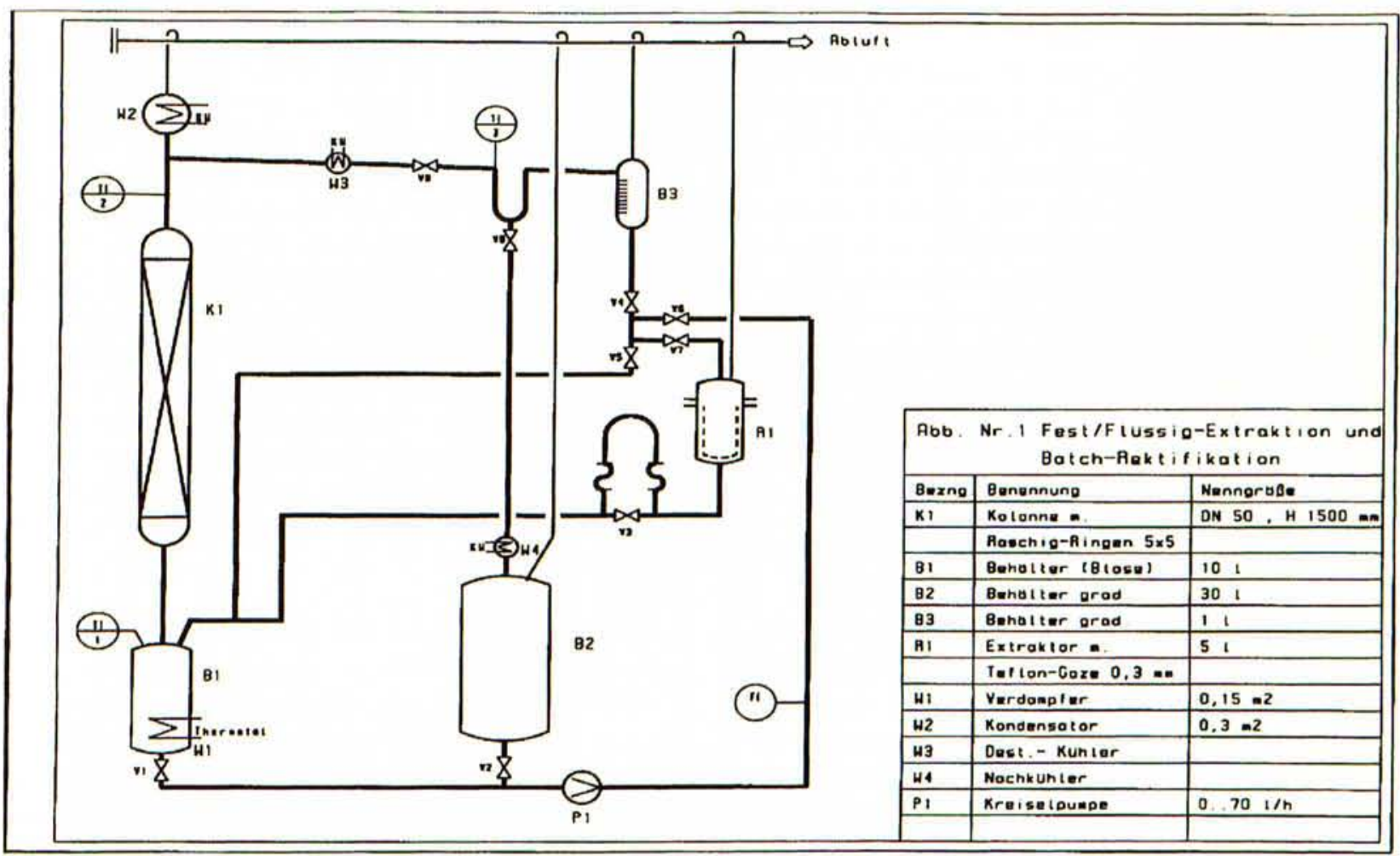

Abb. 2: Verfahrensfließbild der Extraktionsanlage 


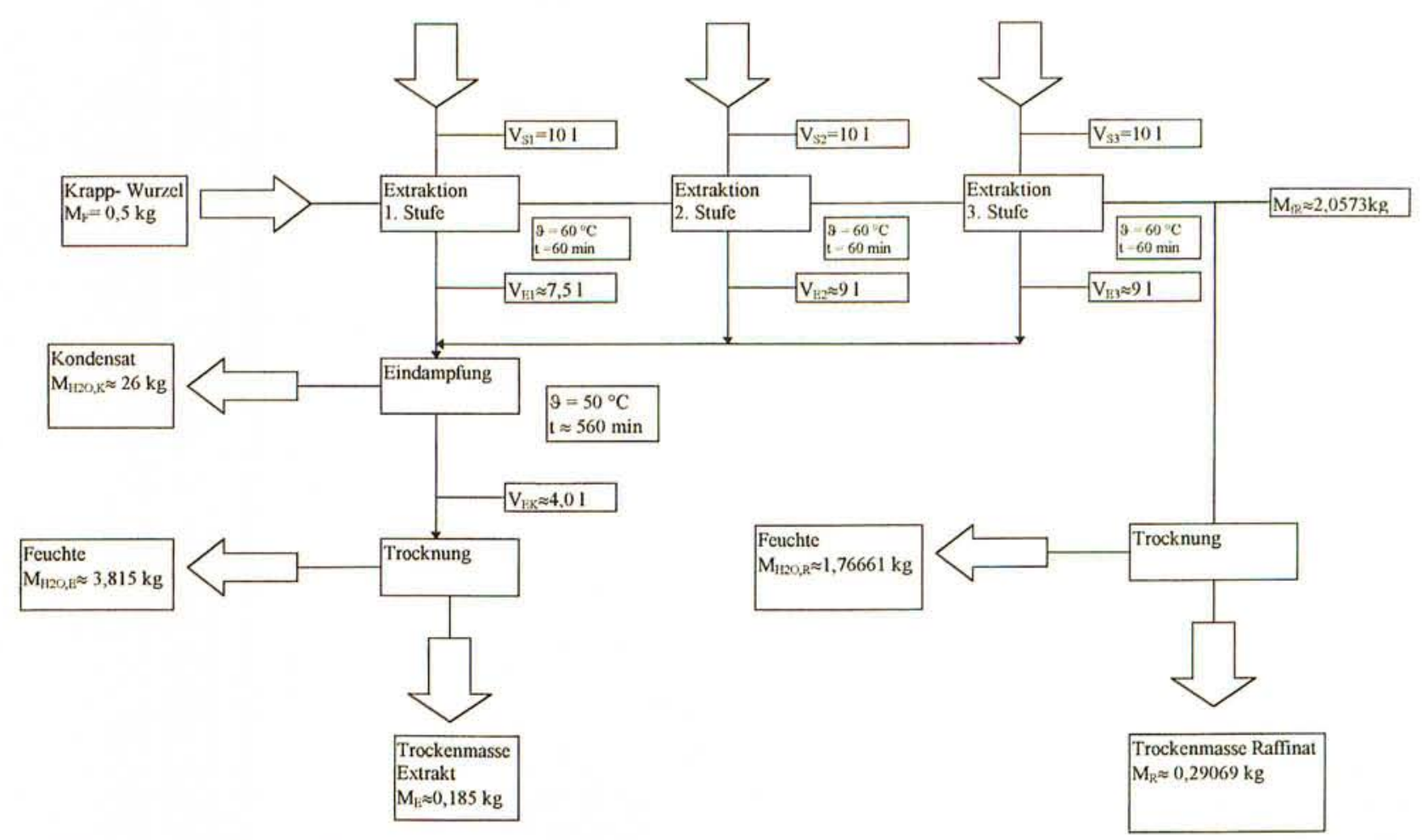

Abb. 3: Typisches Ablaufschema der Versuche

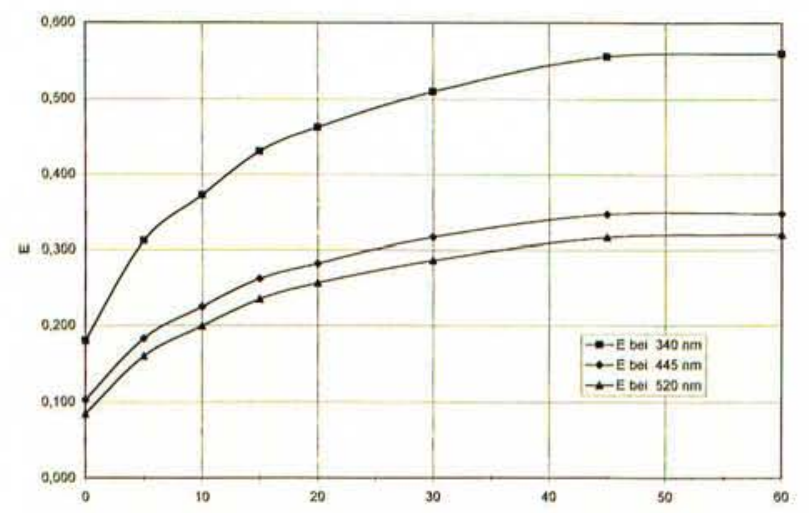

Abb. 4: Abhängigkeit der Extinktion von der Zeit bei drei verschiedenen Wellenlängen für einen typischen Extraktionsversuch.

B-191, teilweise diskontinuierlich in einem Vakuumkammertrockner Typ Heraeus VT6130M-BL und in einem konvektiven Umluftkammertrockner Typ Heraeus UT 6060. Zum Unterschied von der Extraktion ging es dabei nicht um eine systematische verfahrenstechnische Untersuchung der Trocknungsparameter, sondern um die Klärung der prinzipiellen Trocknungsfähigkeit des Krappfarbstoffes unter Beibehaltung der Farbqualität und Vorauswahl eines geeigneten Trocknertyps.

\section{Ergebnisse und Diskussion}

Tabelle 1 enthält eine Übersicht der Versuchsbedingungen für die einzelnen Extraktionsversuche. Es wurden insgesamt 10 Extraktionsversuche bei verschiedenen Temperaturen, in Einzelfällen auch bei gleicher Temperatur und veränderter Stufenzahl, verändertem Lösungsmittelverhältnis oder unter Inertgas durchgeführt. Bis auf Versuch Nr. 1 wurden in allen Fällen $500 \mathrm{~g}$ Krapp-

\begin{tabular}{c|c|r|r|l}
$\begin{array}{c}\text { Versuch } \\
-\mathrm{Nr}\end{array}$ & $\begin{array}{r}\text { Extraktions- } \\
\text { temperatur } \\
{ }^{\circ} \mathrm{C}\end{array}$ & $\begin{array}{r}\text { Extraktions- } \\
\text { mittel- } \\
\text { verhältnis }\end{array}$ & $\begin{array}{r}\text { Stufenzahl } \\
\text { Extraktionszeit } \\
(\mathrm{Npr}=\mathrm{Nth})\end{array}$ & \\
\hline 1 & 75 & $12,5: 1$ & $1 \times 60 \mathrm{~min}$ & $800 \mathrm{~g}$ Wurzelmasse \\
2 & 60 & $20: 1$ & $3 \times 60 \mathrm{~min}$ & \\
3 & 80 & $20: 1$ & $3 \times 60 \mathrm{~min}$ & \\
4 & 75 & $1 \times 12: 1$ & \\
& & $2 \times 20: 1$ & $3 \times 60 \mathrm{~min}$ & Versuch unter $\mathrm{CO}_{2}$ \\
5 & 90 & $20: 1$ & $3 \times 60 \mathrm{~min}$ & \\
6 & 50 & $20: 1$ & $3 \times 60 \mathrm{~min}$ & \\
7 & 60 & $10: 1$ & $6 \times 60 \mathrm{~min}$ & \\
8 & 100 & $20: 1$ & $3 \times 60 \mathrm{~min}$ & \\
9 & 20 & $20: 1$ & $1 \times 225 \mathrm{~min}$ & ohne \\
& & & $1 \times 135 \mathrm{~min}$ & Vorquellung \\
10 & 50 & $20: 1$ & $3 \times 60 \mathrm{~min}$ & \\
\hline
\end{tabular}

Tab. 1: Übersicht der Extraktionsversuche

wurzelmasse eingesetzt, mit der 10 - bis 20 -fachen Extraktionsmittelmenge.

Zunächst war zu klären, wieviele theoretische Extraktionsstufen sinnvoll sind, um eine Extraktionsausbeute von $99 \%$, die industrietechnisch als ausreichend angenommen wird, zu erreichen.

Mit der bekannten Formel aus der Literatur [7] für die theoretische Trennstufenzahl $\mathrm{N}_{\text {th }}$

$$
N_{\text {th }}=\ln f / \ln (1+a)
$$

mit: $f=0,01$, angenommener Extraktionsgrad über alle Stufen [kg Wertstoff im Raffinat der Stufe N]/ [kg Wertstoff im Feed] $\mathrm{a}=4,25$, experimentell ermitteltes Extraktionsmittelverhältnis [kg Solvent im Extrakt der Stufe i]/ [kg Solvent im Raffinat der Stufe i], das mit guter Näherung konstant für alle Stufen angenommen werden darf,

erhält man $\mathrm{N}_{\text {th }}=2,7$. Erforderlich sind demnach 3 theo- 
retische Trennstufen. Diese Überlegung wird bestätigt durch Abb. 5. Darin ist die Extinktion für alle sechs untersuchten Extraktionsstufen des Versuches Nr. 7 in Abhängigkeit von der Extraktionszeit dargestellt. Man beobachtet eine deutliche deutliche Abnahme der Extinktion für die ersten drei Extraktionsstufen. Die Ausgleichskurven furir die 4. bis 6 . Stufe liegen im Rahmen der Fehlergrenze so nahe beieinander, daß sie sich teilweise überschneiden. Aus diesem Grund wurden die Extraktionsversuche in der Regel dreistufig durchgeführt. Die zeitliche Änderung der Extinktion entspricht für die Extraktionsstufen 2. - 6. qualitativ der bereits besprochenen Abb. 4. Lediglich für die 1. Extraktionsstufe sind hier stärkere Streuungen zu beobachten. Diese Streuung der Extinktionswerte trat bei mehreren Versuchen insbesondere während der 1. Extraktionsstufe auf und kann nicht durch Meßungenauigkeiten erklärt werden. Diese Schwankungen können möglicherweise durch die Überlagerung mehrerer Koextraktionsprozesse in Verbindung mit dem zeitlich versetzten Freiwerden von Farbstoffen aus glykosidischen Verbindungen durch Hydrolysereaktionen erklärt werden. Es wird angenommen, daß nichtfärbende Begleitstoffe mit hoher Löslichkeit, wie z. B. Harze, Zucker und Eiweiße besonders in der ersten Stufe extrahiert werden, Alizarin und andere Krappfarbstoffe, die frei oder in glykosidischen Bindungszuständen vorkommen, zu sehr unterschiedlichen Zeitpunkten in Lösung gelangen und dadurch zeitliche Extinktionsschwankungen auftreten, die besonders in der 1. Stufe zu Abweichungen von typischen asymptotischen Anstieg der Extinktion mit der Zeit fuihren.

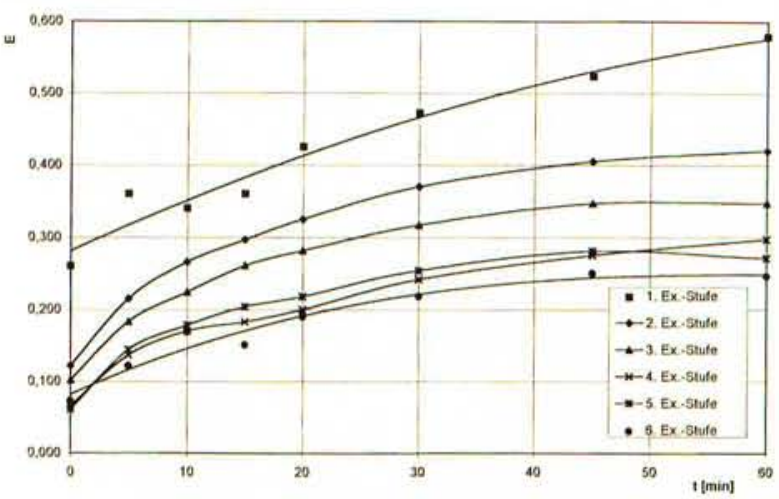

Abb. 5: Abhängigkeit der Extinktion von der Zeit bei einer Wellenlänge von $445 \mathrm{~nm}$ für alle Stufen eines sechsstufigen Extraktionsversuches bei $60^{\circ} \mathrm{C}$.

Generell ist bekannt, daß die Qualität von natürlichen Farbstoffen in der Regel unter der Langzeiteinwirkung hoher Temperaturen leidet. Deren Einwirkungsdauer sollte deshalb minimiert werden. Andererseits begünstigt eine hohe Temperatur die Extraktionsgeschwindigkeit nach den allgemeinen Gesetzen der Stoffübertragung und läßt eine höhere Raum-Zeit-Ausbeute erwarten. Der Einfluß der Temperatur auf die Extraktionsdauer wurde deshalb quantitativ untersucht. Als Extraktionsdauer wurde dabei die Zeit bis zum Erreichen einer theoretischen Extraktionsstufe (Stufenwirkungsgrad $100 \%$ ) betrachtet. In Abb. 6 ist diese Zeit in Abhängigkeit von der Temperatur fuir die jeweils 1. Extraktionsstufe dargestellt. Die Befunde für die 2. und 3. Extrak- tionsstufe sind im Rahmen der Meßgenauigkeit vergleichbar. Man erkennt erwartungsgemäß eine Abnahme der erforderlichen Extraktionszeit mit steigender Extraktionstemperatur. Der Zusammenhang zwischen Extraktionszeit und Temperatur erwies sich dabei annähernd als linear. Sind bei Raumtemperatur noch 80 Minuten bis zum Erreichen der theoretischen Extraktionsstufe erforderlich, so werden bei $100^{\circ} \mathrm{C}$ nur noch 15 Minuten benötigt. Die Rührverhältnisse waren bei allen untersuchten Temperaturen gleich und aufgrund des gewählten hohen Lösungsmitteldurchsatzes $>>$ $100 \mathrm{l} / \mathrm{h}$ annähernd entsprechend der Immersionsextraktion im idealen Rührkessel.

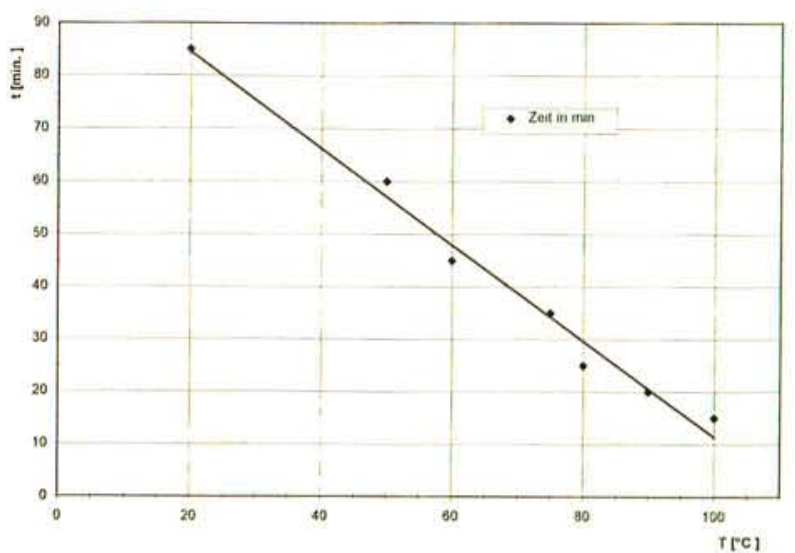

Abb. 6: Abhängigkeit der Extraktionsdauer von der Temperatur.

Aufgrund des in Abb. 4 und Abb. 6 dargestellten Befundes würde man z.B. für eine großtechnische, dreistufige Batch-Extraktion bei $75^{\circ} \mathrm{C}$ - der in alten Färbeanleitungen empfohlenen thermischen Belastungsgrenze - eine Extraktionsdauer von etwa 35 Minuten pro Stufe empfehlen. Bei Betrachtung des gesamten Temperaturbereiches legt das Ergebnis den Schluß nahe, die großtechnische Extraktion, insbesondere dann, wenn sie als Konti- oder Quasikonti-Verfahren (Batch im Wechseltakt) wirtschaftlich sein soll, bei der höchsten Temperatur durchzuführen, um eine hohe Raum-Zeit-Ausbeute und damit eine optimale Wirtschaftlichkeit zu erreichen. Dem steht aber entgegen, daß in traditionellen Färbeanleitungen stets Temperaturen bis $75^{\circ} \mathrm{C}$ empfohlen werden, da bei höheren Färbetemperaturen störende Brauntöne auftreten können. Demnach sind bei Temperaturen $>75^{\circ} \mathrm{C}$ Einbußen bei der Farbqualität zu erwarten. Zur Ermittlung der optimalen Aufarbeitungstemperatur, Extraktionstemperatur eingeschlossen, waren deshalb auch die Extinktionsmessungen und Probefärbungen heranzuziehen. Um diese Frage zu klären, sind in den Abbildungen 7 und 8 jeweils die Extinktion $\mathrm{E}_{\max }-\mathrm{d}$. h. die jeweiligen Höchstwerte der Extinktion gemäß Abb. 4, entsprechend der theoretischen Extraktionsstufe bzw. L"a"b"-Werte von Probefärbungen mit den entsprechenden Extrakten in Abhängigkeit von der Extraktionstemperatur dargestellt.

In Abb. 7 erkennt man, wie erwartet, eine Abnahme der Extinktion $\mathrm{E}_{\max }$ von der 1. zur 3. Extraktionsstufe bei allen untersuchten Temperaturen im Einklang mit Abb. 6, außerdem eine Zunahme mit steigender Tem- 


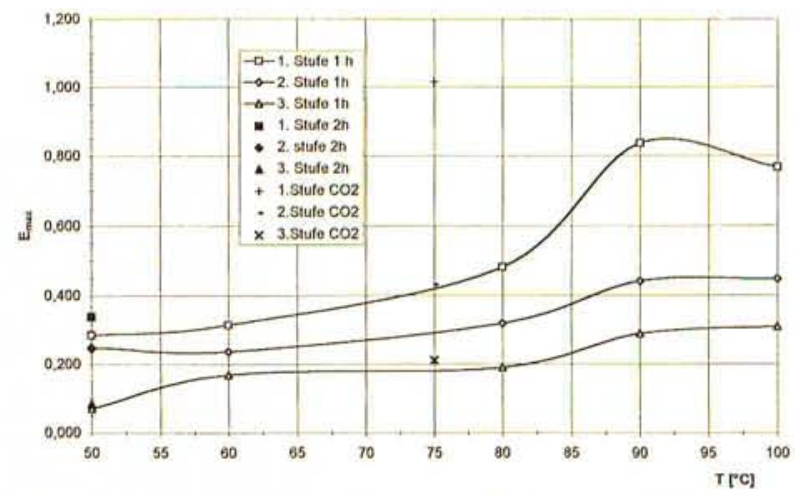

Abb. 7: Abhängigkeit der Extinktion $E_{\max }$ von der Temperatur $T$ bei einer Wellenlänge von $445 \mathrm{~nm}$ für die drei Extraktionsstufen.

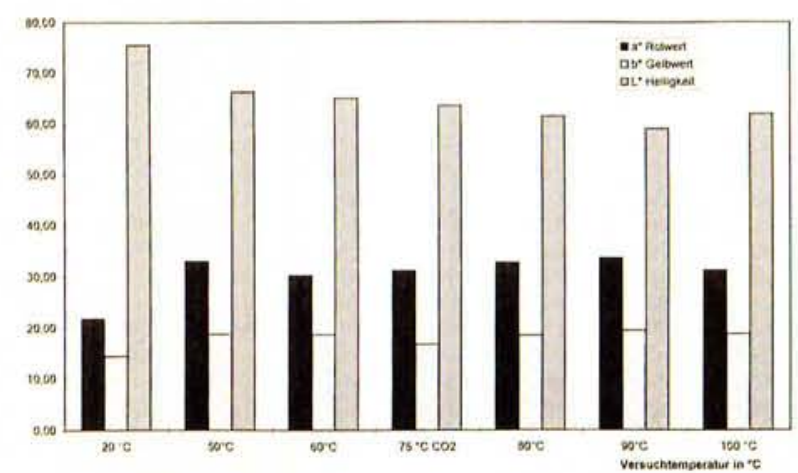

Abb. 8: Farbwerte $a^{\prime}$ (Rot-Grün-Anteil), b' (Gelb-Blau-Anteil) und L' (Helligkeit) in Abhängigkeit von der Extraktionstemperatur.

peratur jeweils für jede Extraktionsstufe bis zu einem Maximum bei $90^{\circ} \mathrm{C}$. Dieser Befund deutet auf eine zunehmende Extraktionsausbeute mit wachsender Extraktionstemperatur hin, wobei oberhalb von $90^{\circ} \mathrm{C}$ möglicherweise Zersetzungsprozesse einsetzen. Entsprechend stellt man in Abb. 8 eine Abnahme der Helligkeit mit der Temperatur fest, während Gelb- und Rotwert mit steigender Temperatur im Rahmen der Meßgenauigkeit nahezu konstant bleiben, sieht man einmal von dem $20^{\circ} \mathrm{C}$-Versuch ab (bei dem möglicherweise das Verteilungsgleichgewicht fest/flüssig bei der Extraktion wegen z. B. adsorptiver Bindungskräfte sehr stark feststofflastig ist). Helligkeit bzw.Farbtiefe und Extinktion korrelieren demnach sehr gut; zunehmende Temperaturen bewirken eine höhere Extraktausbeute entsprechend dem Extinktionsmaximum bei $90^{\circ} \mathrm{C}$ und gleichzeitig eine intensivere Färbung mit einem Minimum der Helligkeit bei derselben Temperatur. Der Vergleich zwischen Extinktion einerseits und Rot- und Gelbwert andererseits läßt aber keinen systematischen Zusammenhang zwischen UV-Absorption und Färbekraft erkennen. Es ist anzunehmen, daß die Extinktion anders als die L"a*b"-Werte nicht nur von den Wirkstoffen des Farbstoffgemisches beeinflußt wird sondern auch von anderen, nichtfärbenden Begleitstoffen, die mitextrahiert werden. Die Abhängigkeit der Rot- und Gelbwerte von der Temperatur zeigt im Gegensatz zur einschlägigen Literatur, daß Temperaturen oberhalb $75^{\circ} \mathrm{C}$ nicht notwendigerweise zu einer Verschlechterung der Färbekraft führen. Erst bei $100^{\circ} \mathrm{C}$ wurde in dieser Arbeit eine Abnahme der Farbintensität gemessen.
Die Aufkonzentrierung der Extrakte, die sich bei allen Versuchen über mehrere Stunden hinzog, fuihrte in der Regel zu einer leichten Abschwächung der Färbekraft [5]. Bei diesem Aufarbeitungsschritt sollten insbesondere Dauerbelastungen von mehreren Stunden oberhalb $60^{\circ} \mathrm{C}$ vermieden werden. Verfahrenstechnisch waren dem Aufkonzentrierungsgrad dadurch Grenzen gesetzt, daß Siedeverzug auftrat und ein weiteres Aufkonzentrieren dadurch nicht möglich war. Für den Vakuumtrockner und den Umlufttrockner waren die dabei maximal erzielbaren Aufkonzentrierungsgrade nicht ausreichend, um in einer vertretbaren Zeit ein brauchbares Trockenprodukt zu erzielen.

Mit einem Labor-Rotationsverdampfer wurde versucht, die Verfahrensschritte Eindampfung und Trocknung zusammenzufassen. Der Zeitaufwand war dabei aber unvertretbar hoch, das Produkt war nicht vollständig trocken und hatte eine pastöse Konsistenz, die eine Weiterverarbeitung sehr erschwerte. Deshalb wurde dieser Verarbeitungsschritt im halbtechnischen Miniplant-Maßstab nicht weiterverfolgt. Hingegen wurden gute Ergebnisse mit dem kontinuierlich arbeitenden Sprühtrockner erzielt. Anders als im Ablaufschema Abb. 3 dargestellt, konnten die Extrakte hier teilweise ohne Vorkonzentrierungsstufe in einem Schritt bis zum Feststoff eingetrocknet werden. Das sprühgetrocknete Produkt war ein feinstkörniges, trockenes und haltbares Pulver mit guter Färbekraft. Weitere Versuche mit Zusatzstoffen, die die Korngröße und Rieselfähigkeit verbessern, scheinen hier sinnvoll, um das Trocknungsverfahren zu optimieren.

\section{Zusammenfassung}

In dieser Arbeit wurde die mehrstufige Kreuzstromextraktion von Farbstoff aus Krappwurzelmaterial mit Wasser als Extraktionsmittel im halbtechnischen Maßstab untersucht und eine Vorauswahl des passenden Trockungsverfahrens getroffen.

Berechnungen und Extinktionsmessungen zu den Extraktionsversuchen zeigten übereinstimmend, daß für eine quantitative Extraktion mit einer Ausbeute von $99 \%$ drei theoretische Kreuzstromstufen ausreichend sind. Die Abhängigkeit der Extraktionsdauer von der Extraktionstemperatur wurde systematisch im Bereich $20-100^{\circ} \mathrm{C}$ untersucht. Extinktionsmessungen und Färbeversuche belegen, daß beim Extrahieren Temperaturen bis zu $90^{\circ} \mathrm{C}$ möglich sind ohne Einbußen in der Farbstoffqualität. Als optimales Lösungsmittelverhältnis kg Extraktionsmittel / kg Wurzelmaterial wurde 20:1 gefunden. Dieser Wert ist aber apparaturspezifisch und kann nicht ohne weiteres auf Apparaturen mit anderen Geometrien übertragen werden. Die Extraktion unter Inertgas mit Kohlendioxid bei $75^{\circ} \mathrm{C}$ brachte keine wesentliche Verbesserung der Farbstoffqualität.

Die Trocknungsversuche ergaben gute Ergebnisse im kontinuierlichen Sprühtrockner, während Trocknungsversuche im Rotationsverdampfer und im Vakuum- und 
Umluftkammertrockner kein konditionierfähiges Produkt lieferten. Das sprühgetrocknete Produkt war trokken und feinstkörnig. Um die Staubverluste im Zyklon des Sprühtrockners zu minimieren, sind weitere Versuche, eventuell mit Zusatzstoffen, die die Korngröße und das spezifische Gewicht erhöhen ohne die Farbqualität zu beeinträchtigen, sinnvoll.

\section{Literatur}

[1] Schweppe, H.: „Handbuch der Naturfarbstoffe. Vorkommen, Verwendung, Nachweis", ecomed-Verlag, Landsberg 1992, S. 231.

[2] Meyer, E.: „Erzeugung und Anwendung von pflanzlichen Farbstoffen für die Textilfärbung“, Forschungsprojekt, MELF-Brandeburg (Kapitel 10031 - Titel 68564), Arbeitsbericht des IfU Schlieben 1997.

[3] Fischer, C; Bischof, M. und Rabe, J. G.: „Identification of natural and early synthetic textile dyes with HPLC and UV/ VIS-spectroscopy by diode array detection", J. of Liquid Chromatography Nr. 13 (1990), S. 319-331.

[4] Blaß, E., Liebl, T. und Häberl, M.: „Extraktion, ein historischer Rückblick“, Jahrbuch 1997 Verfahrenstechnik und Chemieingenieurwesen, VDI-Verlag GmbH Düsseldorf, 1997, S. $117-133$.

[5] Nencki, Z. B. L.: „Die Kunst des Färbens mit natïrlichen Stoffen“, Haupt-Verlag Bern und Stuttgart, 1984.

[6] Abramowski, S.: „Extraktion und Trocknung von Inhaltsstoffen der Krappwurzel“, Diplomarbeit Technische Fachhochschule Wildau, 1998 (in Vorbereitung).

[7] Vauck, W. R. A., Müller, H. A.: „Grundoperationen chemischer Verfahrenstechnik“, 9. Auflage, Dt. Verlag für Grundstofffind., Leipzig, 1992.

\section{Verfasser}

Prof. Dr.-Ing. Josef Schmadl

cand. ing. Stephan Abramowski

Technische Fachhochschule Wildau

Fachbereich Ingenieurwesen/Wirtschaftsingenieurwesen

Tel. (0 33 75) 507-110, -177

E-mail: jschmadl@igw.tfh-wildau.de

\section{Dr. Eckhard Meyer}

Institut für Umweltforschung Schlieben e.V.

Gartenstraße 43, 04936 Schlieben

Tel. (03 53 61) 534, Fax (03 53 61) 722 